\title{
1
}

\section{Colloidal Lubrication: General Principles}

\author{
Jean Michel Martin and Nobuo Ohmae
}

Nanoparticles can be considered as potential lubricant additives. They present several major advantages compared to organic molecules currently used as lubricant additives, for example:

- Their nanometre size allows them to enter the contact area easily.

- They are often efficient at ambient temperature. Thus, no induction period is necessary to obtain interesting tribological properties.

- Several types of nanoparticles can be envisaged as lubricant additives. The chapters of this book describe different possible nanolubricants: nested nanoparticles (fullerenes, onions and nanotubes) made on metal dichalcogenides or carbon, reverse overbased micelles, metallic nanoparticles and boron-based nanolubricants. In some cases, the authors chose to study these particles because their structure is close to well-known lamellar structures in tribology (for example inorganic fullerenes of $\mathrm{MoS}_{2}$ ). However, dispersion of nanoparticles in oil is not always simple. Thus, in a first part, some rules will be shown about the dispersion of solid nanoparticles in a liquid.

Another way to use nanoparticles in tribology is by embedding them inside a coating. Nanoparticles can improve the tribological of the coating itself, but nanoparticles can also be released by the coating during wear and can thus lubricate in the same way as if they were in the oil.

\subsection{Stability of Colloids Dispersed in a Base Oil}

A colloidal sol is generally a substance (liquid or gel) containing dispersed solid particles. To obtain a homogeneous and stable system, the size of the nanoparticles has to be small, say below $100 \mathrm{~nm}$.

Nanolubricants Edited by J. M. Martin and N. Ohmae

(C) 2008 John Wiley \& Sons, Ltd 


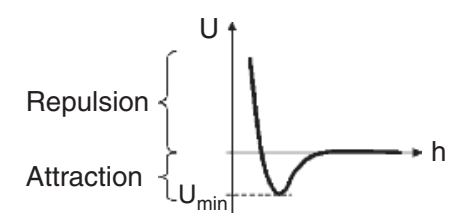

Figure 1.1 Lennard-Jones force describing attraction and repulsion between two molecules as a function of the distance between them

A sol is composed of two phases: a continuous phase and a dispersed phase. In the present case, the continuous phase is liquid (oil) and the dispersed phase is a solid (nanoparticles). A good stability of dispersion is not easy to obtain, and several phenomena lead to instability:

- aggregation (or flocculation) of the particles;

- sedimentation of particles and aggregates of particles.

Lennard-Jones forces are often used to describe intermolecular interactions. They result from steric repulsion at very short distance and attractive van der Waals forces at short distance (Figure 1.1).

At a short distance (below $50 \mathrm{~nm}$ ), two particles attract themselves due to van der Waals forces. This force is expressed by the following relation:

$$
E_{\mathrm{vdw}}=-\frac{A_{r}}{12 h}
$$

where $A_{r}$ is the Hamaker constant and $h$ is the distance between particles. The Hamaker constant is equal to $\pi^{2} C \rho^{2}$ ( $C$ is a constant that is characteristic of the material constituting the particles and of the dispersion medium). These forces become more significant when the distance $h$ is below $10 \mathrm{~nm}$.

Particles in suspension in a liquid medium are subjected to three kinds of forces: (a) gravitation forces the particles to fall down, (b) viscosity of the liquid decreases the speed of their displacement and (c) Archimedes force is opposed to gravitation forces in this case. By applying fundamental relation of dynamics, the expression of the steady state sedimentation speed of a particle is obtained:

$$
V=\frac{2}{9} \frac{R^{2}}{\eta}\left(\rho-\rho^{\prime}\right) g
$$

with

$R$ radius of the particle

$\eta$ dynamic viscosity of the liquid

$\rho$ density of the particles

$\rho^{\prime}$ density of the liquid 
It is important to notice that the speed varies in proportion to the square of the radius of the particle. Therefore, particles with large radius will sediment much faster than small ones. Due to the flocculation process described before, aggregated particles will present a larger size than individual ones and sedimentation is then faster.

Fortunately, other effects can allow for a better stability of a suspension. The Brownian motion is certainly the most interesting. It corresponds to chaotic movement of particles due to shocks of liquid molecules on its surface. For small particles, the relation of Stokes-Einstein gives the average distance $x$ covered by a particle during a certain time $t$ as

$$
x=\sqrt{\frac{2 k T t}{6 \pi \eta R}}
$$

with

$R$ radius of the particle

$\eta$ dynamic viscosity of the liquid

$T$ temperature

$k$ constant of Boltzmann $\left(k=1.38 \times 10^{-23} \mathrm{~J} / \mathrm{K}\right)$

$t$ time

Some nanoparticles are difficult to disperse in oil. For example, inorganic fullerenes (studied in Chapter 2) are mineral particles with a spherical or cylindrical shape and a size lying between 50 and $150 \mathrm{~nm}$. They do not present any affinity with oil and their dispersion is thus difficult without surfactants.

To study the dispersion of such nanoparticles in base oil, they were mixed at different concentrations in poly-alpha-olefin (PAO) base oil using an ultrasonic bath (concentrations are expressed here in weight percent). By using formulae (2) and (3), the distance covered by one particle in $1 \mathrm{~s}$ can easily be compared by sedimentation and by Brownian motion, respectively (Table 1.1). The sedimentation force is oriented vertically whereas the distance covered by Brownian movement has no preferential direction. For this calculation, the density of the lamellar compounds was fixed at $5.06 \mathrm{~g} / \mathrm{cm}^{3}$ for $\mathrm{MoS}_{2}, 7.6 \mathrm{~g} / \mathrm{cm}^{3}$ for $\mathrm{WS}_{2}$ and $4.4 \mathrm{~g} / \mathrm{cm}^{3}$ for $\mathrm{NbS}_{2}$ (according to the data from the supplier). The value used for inorganic fullerene (IF)

Table 1.1 Dispersion properties of IF-MS 2 and corresponding lamellar structures at $20^{\circ} \mathrm{C}$

\begin{tabular}{|c|c|c|c|}
\hline & $\begin{array}{l}\text { Average diameter } \\
(\mathrm{nm})\end{array}$ & $\begin{array}{c}\text { Sedimentation: } \\
\text { distance covered in } \\
1 \mathrm{~s}(\mathrm{~nm})\end{array}$ & $\begin{array}{l}\text { Brownian } \\
\text { movement in } \\
1 \mathrm{~s}(\mathrm{~nm})\end{array}$ \\
\hline \multirow[t]{2}{*}{ IF-WS ${ }_{2}$} & 140 & 0.2 & 150 \\
\hline & 90 & 0.1 & 188 \\
\hline $2 \mathrm{H}-\mathrm{WS}_{2}$ & 1000 & 55 & 39 \\
\hline IF-MoS 2 & 40 & 0.01 & 282 \\
\hline $2 \mathrm{H}-\mathrm{MoS}_{2}$ & 2000 & 140 & 28 \\
\hline IF-NbS ${ }_{2}$ & 60 & 0.02 & 230 \\
\hline 3R-NbS 2 & 1000 & 29 & 13 \\
\hline
\end{tabular}


Table 1.2 Dispersion properties of IF-MS 2 and corresponding lamellar structures at $70{ }^{\circ} \mathrm{C}$

\begin{tabular}{lccc}
\hline & $\begin{array}{c}\text { Average diameter } \\
(\mathrm{nm})\end{array}$ & $\begin{array}{c}\text { Sedimentation: } \\
\text { distance covered } \\
1 \mathrm{~s}(\mathrm{~nm})\end{array}$ & $\begin{array}{c}\text { Brownian } \\
\text { movement in } \\
1 \mathrm{~s}(\mathrm{~nm})\end{array}$ \\
\hline $\mathrm{IF}^{-W S_{2}}$ & 140 & 2 & 503 \\
& 90 & 0.9 & 628 \\
$2 \mathrm{H}-\mathrm{WS}_{2}$ & 1000 & 531 & 133 \\
$\mathrm{IF}-\mathrm{MoS}_{2}$ & 40 & 0.1 & 942 \\
$2 \mathrm{H}-\mathrm{MoS}_{2}$ & 2000 & 1329 & 94 \\
$\mathrm{IF}-\mathrm{NbS}_{2}$ & 60 & 0.2 & 770 \\
$3 \mathrm{R}-\mathrm{NbS}_{2}$ & 1000 & 280 & 133 \\
\hline
\end{tabular}

is $15 \%$ lower than in the $2 \mathrm{H}$ structure to take into account the cavity generally present in the centre of IF particles.

The results show the advantage of using smaller IF-MS 2 particles instead of the corresponding $2 \mathrm{H}$ structure. The sedimentation speed is much higher in the case of the lamellar structure (which is a few tenths of a micrometre in size). Thus, calculations predict much better stability of IF suspensions, the distance due to sedimentation being lower than that of the Brownian motion. However, flocculation of particles could explain the poor stability of the suspensions because of the absence of any polar molecules. Calculations show that an aggregate of particles with a diameter of $650 \mathrm{~nm}$ will cover about the same distance by sedimentation and by Brownian motion.

An increase in the temperature provides a beneficial effect on the increased Brownian motion. However, it drastically decreases the viscosity of the oil and thus sedimentation of particles becomes faster. A comparison of results in Tables 1.1 and 1.2 illustrates this phenomenon, which is of course more important in the case of larger particles.

The stability of dispersions of PAO + IF - $\mathrm{MS}_{2}$ was determined optically by measuring the time necessary to observe the total sedimentation of fullerenes at $20^{\circ} \mathrm{C}$ (Table 1.3). These results confirm the importance of the size of the particle on the stability of the dispersion. The best stability is obtained for the oil/IF-MoS 2 dispersion, having a smaller particle size (about $40 \mathrm{~nm}$ ).

In the case of nanotubes, the problem of dispersion is more complex and there are no simple relations to express the various trends. Generally nanotubes are intermingled and tend to form bundles, leading to a very difficult dispersion. The length of the nanotubes is certainly a crucial parameter.

Table 1.3 Sedimentation times for different nanoparticle dispersions at $20^{\circ} \mathrm{C}$. The times were determined optically

\begin{tabular}{lcccc}
\hline & $\begin{array}{c}\mathrm{IF}^{-M o S_{2}} \\
(40 \mathrm{~nm})\end{array}$ & $\begin{array}{c}\mathrm{IF}-\mathrm{NbS}_{2} \\
(60 \mathrm{~nm})\end{array}$ & $\begin{array}{c}\mathrm{IF}^{2}-\mathrm{WS}_{2} \\
(90 \mathrm{~nm})\end{array}$ & $\begin{array}{c}\mathrm{IF}^{2}-\mathrm{WS}_{2} \\
(140 \mathrm{~nm})\end{array}$ \\
\hline $\begin{array}{l}\text { Sedimentation } \\
\text { time (hours) }\end{array}$ & 10 & 8 & 6.5 & 5.5 \\
\hline
\end{tabular}


Stability of a colloidal solution can be improved:

- by using steric repulsion between particles: functionalization by polymers;

- by using electrostatic repulsion: adsorption of ionic surfactants.

In the case of nanotubes, several techniques were proposed: functionalization by organic molecules [1] and the use of surfactants like sodium dodecyl sulfate (SDS) to disperse them in water. This kind of dispersion led to the manufacture of fibres made of nanotubes [2]. Nanotubes can also be cut to avoid their intermingling and thus improving their dispersion.

For the dispersion of IF-WS 2 , a technique using small metallic balls has been developed by Nanomaterials Company (http://www.apnano.com). Vigorous stirring by using small metal balls makes it possible to obtain better dispersions compared with an ultrasonic bath. Moshkovith et al. studied the dispersion parameters on the tribological properties of IF-WS and, in particular, the size of aggregates (large aggregates $>2 \mu \mathrm{m}$ and small aggregates $<1 \mu \mathrm{m})$ as a function of the mixing time of the dispersion [3]. The increase of the mixing time leads to a decrease in the aggregate size. Friction results are then more reproducible.

\subsection{Lubrication by Micellar Systems}

Detergents in oils form micelles, which are aggregates of surfactant molecules. Polar molecules are made of a polar part (hydrophilic head) and a nonpolar hydrophobic part. According to the nature of the solvent (polarity), they can form micelles or reverse micelles. Hydrocarbon chains create a steric barrier, preventing the particles from aggregation. Overbased micelles have a mineral core made, for example, of calcium carbonate $\mathrm{CaCO}_{3}$ (Figure 1.2).

Owing to their lipophilic chains and their very small size, micelles are very easily dispersed in base oils and dispersion is stable for a long time. Overbased micelles, used initially as detergents, also present antiwear properties (see Chapter 4). Overbased micelles can be used in several ways: (a) as thin coatings deposited on a solid surface, (b) as a colloidal dispersion in apolar medium (oil, grease, etc.) and (c) in gas phase lubrication.

The ability of overbased micelles to lubricate to some extent compared with molecules can be explained on the basis of the following features:

1. Micelles can adsorb physically on any surface (metal, ceramics, carbon-rich materials, etc.) because physical laws (van der Waals forces) mainly govern the attractive forces. In particular, they can adsorb at low temperature.

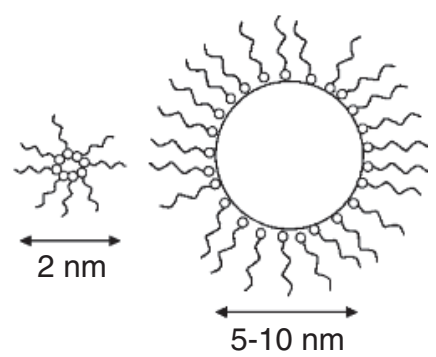

Figure 1.2 Schematic representation of an inverse micelle and overbased micelle structure 
2. The adsorbed micelles are so small that they can hardly be removed from the surface by the moving liquid.

3. The size of the micelles is so small that they can easily enter a macroscopic sliding contact in boundary conditions but do not perturb the hydrodynamic regime significantly.

4. The lubrication effect can be generated by the chemical nature of both constituents of the micelles (the core and the shell) and even by the synergistic effect between the two.

Moreover, tribochemical reactions in the contact area can create a new compound as the third body.

Overbased micelles are generally obtained by a polyphasic complex reaction. They are prepared by carbonating a metal or by hydroxide, which is dispersed in a polar mixture of surfactant, aromatic solvant and paraffinic oil [4,5]. Prior to bubbling carbon dioxide through the reaction vessel, polar promoters (alcohols, amines and water) are added. Thus, oxide or hydroxide particles can be converted into spherical colloidal particles in the size range of $2-20 \mathrm{~nm}$. The colloidal dispersion obtained is centrifuged (typically 30 minutes at $3000 \mathrm{~g}$ ) in order to eliminate nonreacted micrometre-sized lime particles and large aggregates of crystallized micelles. Afterwards, the colloidal suspension is dialysized using pure heptane, to remove organic impurities and nonreacted micelles. At the end of dialysis, the heptane is evaporated and a yellow-brown solid varnish-like material is obtained, which can be stored and further used for any lubrication mode. The structure of the solid phase has been determined by small-angle electron diffraction in transmission electron microscopy (TEM) (see Figure 1.3). Preparation of a coating can be made using the standard procedure for LangmuirBlodgett films. A monomicellar film can be produced on water and then transferred to a surface by dipping. This shows the amorphous character of the structure at the micellar level and the fact that colloids are packed together with the original structure they originally had in the solution (Figure 1.3). Another technique simply uses solvent evaporation after deposition of a drop of the solution on the surface. However, in this case, the thickness of the coating is not controlled. The fixation of micelles on surfaces has been attributed to van der Waals forces between the lipophilic end of surfactant chains and the solid surface, and also to the attraction of the mineral core by the substrate. The attractive forces as shown by TEM observation can

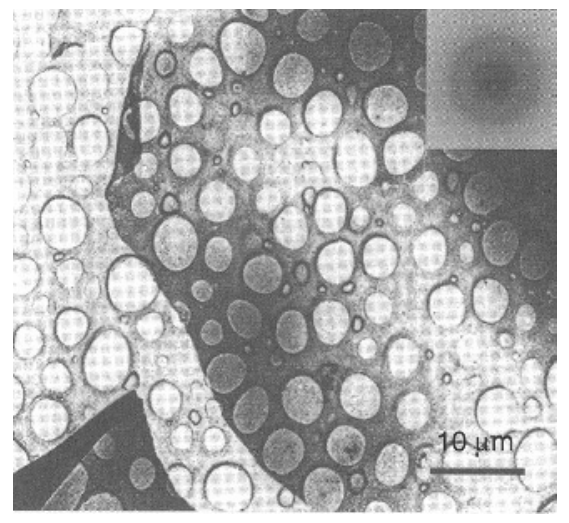

Figure 1.3 TEM image of Langmuir film made of overbased micelles 
(a)

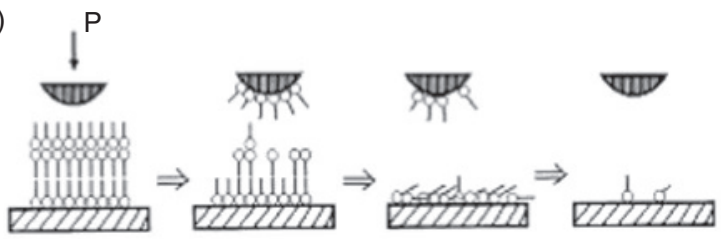

(b)

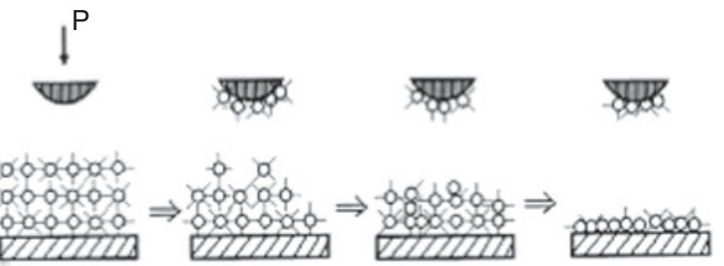

Figure 1.4 Lubrication mechanism of (a) fatty acid LB films and (b) $\mathrm{MoS}_{2}$-DDP LB films. Reproduced by permission of Elsevier from Zhang et al. [8], (c) (2000) Elsevier

mechanically deform the mineral core when a film of micelles is deposited on a carbon film. Overbased micelles can be directly used as additive in a base oil. Due to the small size of the nanoparticle and the presence of the surfactant, the dispersion is very stable. Lubrication by such micellar systems are described in Chapter 4.

\subsection{Lubrication by Metallic Nanoparticles}

Metal nanoparticles are not easily dispersed in oil unless that they are of nanometre size. Zhou et al. studied the tribological properties of $\mathrm{Cu}$ nanoparticles dispersed in pure paraffin base oil [6]. To improve their compatibility with the base oil, the surface of $\mathrm{Cu}$ nanoparticles was modified with dialkyldithiphosphate (DDP). Zhang et al. used the same surface modification process with Mo-S nanocluster [7]. These nanoparticles can be used as additives in lubricant or as Langmuir-Blodgett (LB) films [8]. Zhang et al. compared the tribological behaviour of LB films of fatty acid and $\mathrm{MoS}_{2}$-DDP (Figure 1.4). $\mathrm{MoS}_{2}$-DDP films present better antiwear properties. These properties can be explained by a higher load-carrying capacity of $\mathrm{MoS}_{2}$ nanocores. Chapter 5 is dedicated to metallic nanolubricants.

\subsection{Colloids Embedded in a Coating}

Nanoparticles can also be used as coatings or embedded in coatings. IF-MS ${ }_{2}, \mathrm{C}_{60}$ and carbon nanotubes can be added into coatings that present interesting properties. Chhowalla and Amaratunga compared thin IF-MoS ${ }_{2}$ films $\left(1 \mu \mathrm{m}\right.$ thick) and $2 \mathrm{H}-\mathrm{MoS}_{2}$ films, and found a very low friction coefficient with IF-MoS $\mathrm{S}_{2}$ coatings, even in $\mathrm{N}_{2}$ atmosphere [9]. These performances were explained by the absence of edges due to the nested structure of fullerenes. Joly-Pottuz compared the tribological properties of IF-MoS 2 and IF-WS 2 films, obtained by solvent evaporation or rubbing, under ultrahigh vacuum. Very low friction coefficients were obtained with all coatings (see Chapter 2). Recently, IF-WS 2 particles were deposited on diamond-like carbon (DLC) coatings and very a low friction coefficient was observed [10]. 


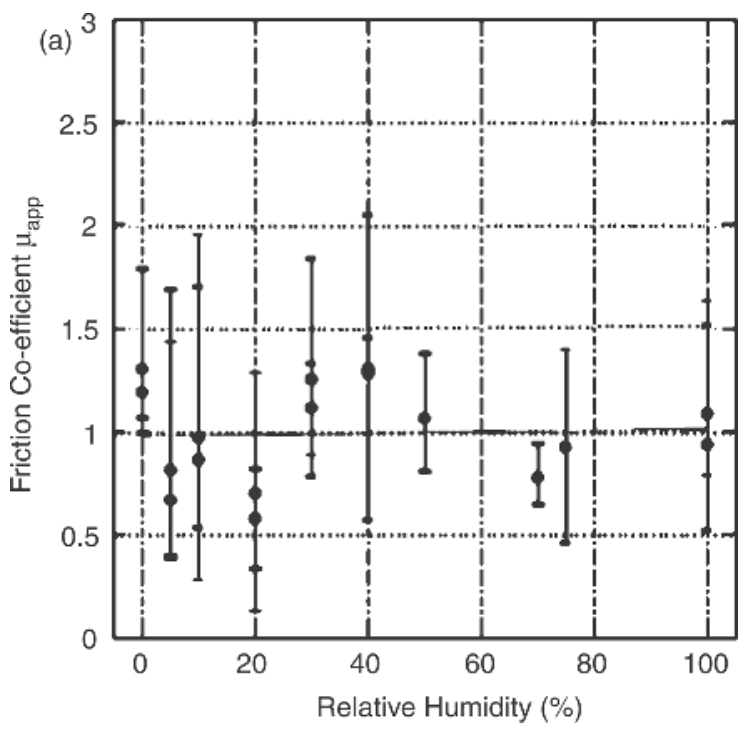

Figure 1.5 Influence of humidity on the friction coefficient of carbon nanotube film. With kind permission from Springer Science and Business Media from Turq et al. [14], (c) (2005) Springer Science and Business Media

Nakagawa et al. used molecular beam epitaxy (MBE) to deposit very thin coatings of $\mathrm{C}_{60}$. Experiments by friction force microscopy (FFM) reveal a very low friction force [11]. Carbon nanotubes can be grown on surfaces to form film usually called 'forest' [12]. These films present particular properties. They are highly hydrophobic with a contact angle of $162^{\circ}$. The frictional behaviour of these films was evaluated against a gold tip [13]. High friction coefficients (1.2 to 1.5$)$ were measured but no adhesion of the tip was observed. These properties can be attributed to the deformation properties of these films. Their deformation was followed by using a tribometer inside a scanning electron microscope (SEM). The influence of humidity on their tribological properties showed that friction is independent of the relative humidity (Figure 1.5) [14]. Miyoshi et al. also studied friction properties of multiwall carbon nanotube (MWNT) coatings. Friction coefficients of aligned MWNTs against steel were measured between 0.025 and 0.06 in air, and between 0.19 and 0.48 in vacuum [15].

Coating structures have changed during the last 25 years. A recent paper by Donnet and Erdemir gives an overview of the evolution of coatings [16]. Coatings are now multicomponent (three, four or more phases) and/or multilayered. These evolutions lead to an optimization of their properties: antiwear resistance and corrosion resistance. Addition of nanoparticles in coatings was also found to improve their properties. For example, addition of $\mathrm{Al}_{2} \mathrm{O}_{3}$ nanoparticles in polymer coatings leads to an improvement in their scratch and abrasive resistances [17].

Inorganic fullerenes of $\mathrm{MoS}_{2}$ have been also added into coatings. Chen et al. compared tribological results obtained with Ni-P electroless composite coatings containing $2 \mathrm{H}-\mathrm{WS}_{2}$, graphite and IF-WS $\mathrm{WS}_{2}$ [18]. Addition of graphite or $2 \mathrm{H}-\mathrm{WS}_{2}$ leads to a reduction of the friction coefficient, 0.067 and 0.062 , respectively, compared to the one obtained for pure Ni-P coating (0.09). However, addition of IF-WS 2 leads to a strong friction reduction (0.03). No wear was observed on the Ni-P-(IF-WS2) coatings after friction tests (only slight scars). On the 


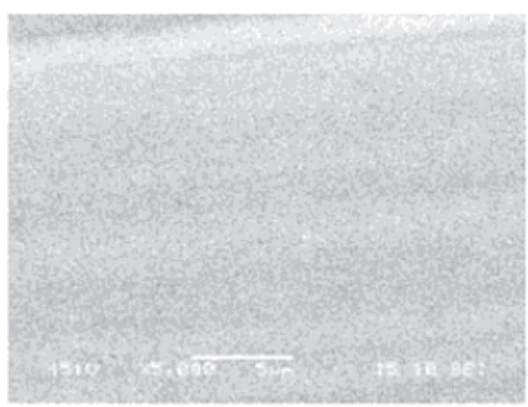

(a) Ni-P-(IF-WS 2 ) coating

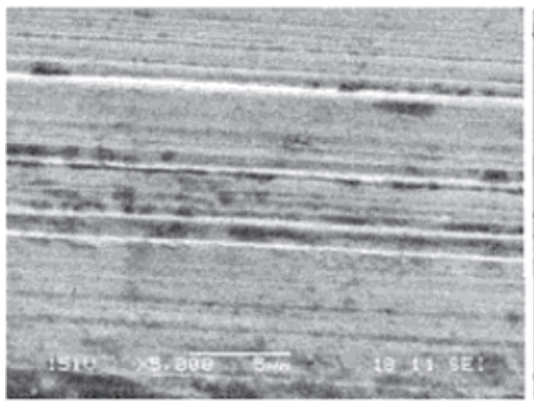

(c) Ni-P-graphite coating

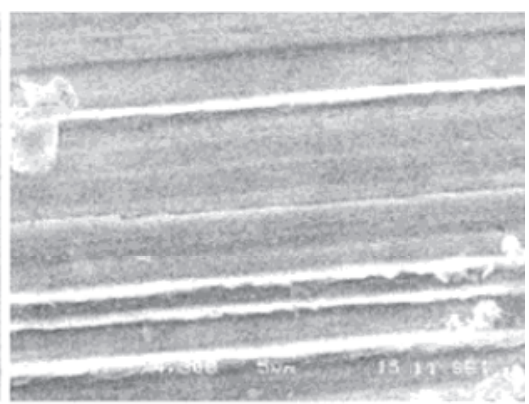

(b) Ni-P-(2H-WS $)_{2}$ coating

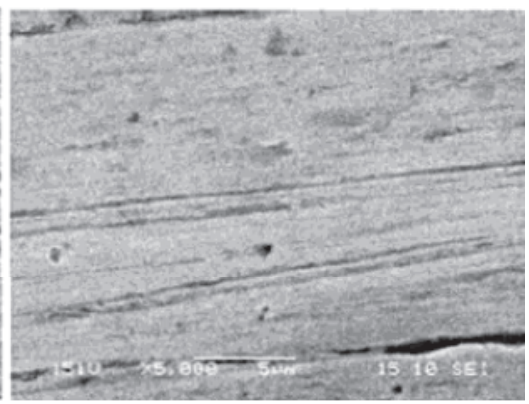

(d) Ni-P coating

Figure 1.6 SEM images of worn surface of Ni-P coatings (d) and Ni-P coatings containing (b) $2 \mathrm{H}-$ $\mathrm{WS}_{2}$, (c) graphite or (a) IF-WS 2 [18]

contrary, wear was observed with the three other coatings (see Figure 1.6). Interesting results were also observed with Ni-P-(IF-MoS $)_{2}$ coatings tested in air and vacuum, while Ni-P- $(2 \mathrm{H}-$ $\mathrm{WS}_{2}$ ) presented a poor stability [19]. Katz et al. added IF-WS 2 nanoparticles in Ni-P coatings to lubricate orthodontic wires [20]. These coatings are envisaged to lubricate other medical devices (catheter and endoscope).

Friction results can not be explained by a rolling process of the fullerenes. Indeed, nanoparticles embedded in the coatings are unable to roll [21]. Rapoport et al. studied tribological properties of polymer nanocomposites with embedded IF nanoparticles [22]. The friction mechanism was found to be due to a transfer of IF from the polymer coatings to the counterface. The same mechanism was proposed for bronze powder composites impregnated with nanoparticles [23]. Coatings impregnated with IF-WS 2 present better properties (the transition to seizure is higher) than $2 \mathrm{H}-\mathrm{WS}_{2}$ impregnated ones. Platelets of $2 \mathrm{H}-\mathrm{WS}_{2}$ can not easily enter into the pores of the coatings and are accumulated at the metal surface. On the contrary, fullerenes enter the pores of the coatings easily and are gradually supplied inside the contact area. Thus, their effect on the tribological properties of the coatings is made longer. These two examples demonstrate that fullerenes containing coatings act by progressively releasing the particles in the contact area.

Nanotubes present exceptional mechanical properties and are now widely used to reinforce composites [24], particularly with polymers [25]. Tribological properties of composites 


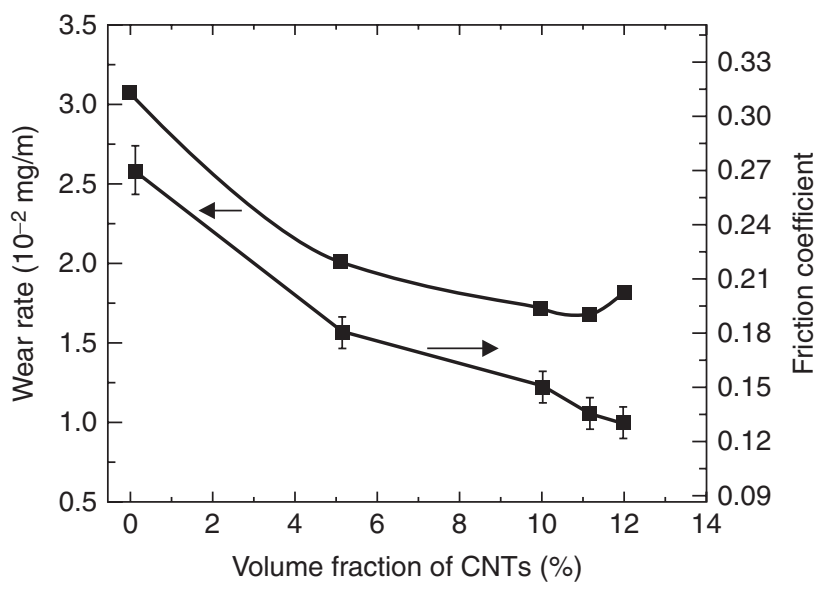

Figure 1.7 Variation of friction coefficient and wear loss of Ni-based composite with volume of carbon nanotubes embedded in the coatings. Reproduced by permission of Elsevier from Wang et al. [27], (C) (2003) Elsevier

containing nanotubes have also been widely studied. Several matrices can be considered: polyimide [26], Ni [27], Ni-P [28] and alumina [29,30]. A general trend is observed for all these matrices: the friction coefficient and the wear loss decreases when the percentage of carbon nanotubes in the coatings increases, as presented in Figure 1.7 for Ni-based carbon nanotube (CNT) composite. In the case of carbon/carbon composite, the addition of carbon nanotubes leads to a reduction in wear loss, but the friction coefficient slightly increases with the percentage of carbon nanotubes in the coatings [31].

The dispersion of nanotubes inside the matrix is not always easy. To solve this problem, Schittelhelm et al. proposed to deposit ta-C coatings by physical vapour deposition (PVD) on nanotubes previously deposited on $\mathrm{Si}$ substrates [32]. This paper shows that the amorphous diamond film provides scratch resistance for dispersed single-wall nanotubes (SWNTs). This technique can be envisaged to grow thin films containing carbon nanotubes. Recently, DLC coatings were also deposited on carbon nanotube forests (see Figure 1.8). Mechanical properties of these coatings were studied against the density of carbon nanotube forests ( 1 to $7 \times 10^{9} / \mathrm{cm}^{2}$ ). The dynamic hardness and elastic modulus of the CNT/DLC composite films

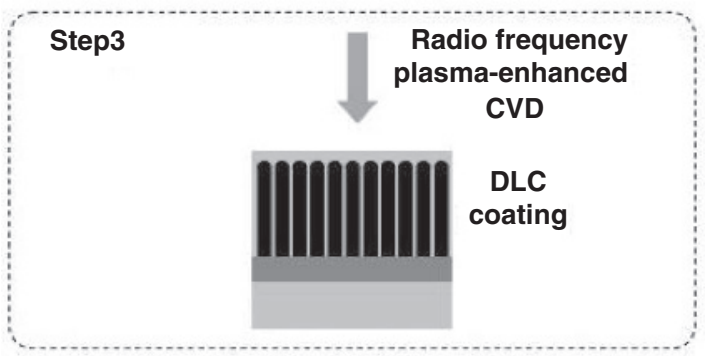

Figure 1.8 Schematic composition of CNT/DLC coatings [33] 


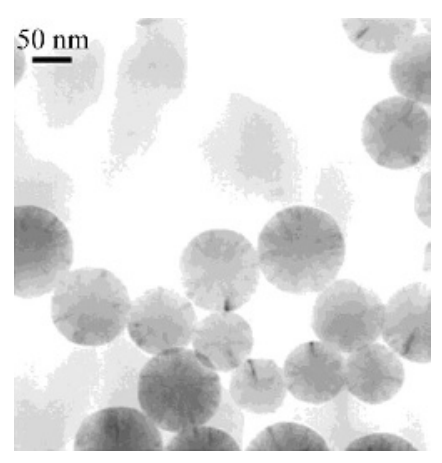

Figure 1.9 TEM image of carbon onions randomly distributed on a graphite film. Reproduced by permission of Elsevier from Cabioc'h et al. [36], (c) (2000) Elsevier

decrease linearly with increasing CNT density, because the DLC coating has a much higher hardness and elasticity than CNT films. The same friction coefficient of 0.23 was observed and was independent of the density of nanotubes. This friction coefficient corresponds to those obtained with pure DLC coatings (not deposited on CNT forests).

A way to synthesize carbon onions is by high-fluence ion implantation of carbon onions into metals $(\mathrm{Cu}, \mathrm{Ag})$. This is possible because of the very low solubility of carbon into copper or silver. The implanted carbon atoms precipitate into the bulk of the metal. A uniform graphite layer is formed with the carbon onions above [34]. Figure 1.9 presents a film observed after ion implantation of copper. Implantation parameters have an influence on the size of carbon onions: the mean diameter is $5 \mathrm{~nm}$ for a dose of $5 \times 10^{16} / \mathrm{cm}^{2}$ and $20 \mathrm{~nm}$ for $2.5 \times 10^{17} / \mathrm{cm}^{2}$ [35]. The growth mechanism of the graphite film and carbon onions was proposed by Cabioc'h et al. [34]. Tribological properties of these carbon films containing carbon onions were tested (after removal of silver by thermal annealing at $850{ }^{\circ} \mathrm{C}$ ). Unfortunately, their thickness was too thin [37]. However, silver films containing carbon onions were tested, and friction results obtained were compared with those obtained on pure silver films. The presence of onions in silver films leads to an increase in lifetime of the coating by a factor of 15 .

During the preparation of this chapter, such a new finding that carbon onion synthesized from diamond nanoparticle at $1600{ }^{\circ} \mathrm{C}$ shows a low friction coefficient of 0.005 in ultrahigh vacuum. Therefore, further application of nanolubricants to tribology is anticipated.

\section{References}

1. Chen, J., Hamon, M. A., Hu, H., Chen, Y. A., Rao, M., Eklund, P. C. and Haddon, R. C., Solution properties of single-wall carbon nanotubes, Science, 282, 1998, 95-98.

2. Vigolo, B., Pénicaud, A., Coulon, C., Sauder, C., Pailler, R., Journet, C., Bernier, P. and Poulin, P., Macroscopic fibers and ribbons of oriented carbon nanotubes, Science, 290, 2000, 1331.

3. Moshkovith, A., Perfiliev, V., Verdyan, A., Lapsker, I., Popovitz-Biro, R., Tenne, R. and Rapoport, L., Sedimentation of IF-WS ${ }_{2}$ aggregates and a reproducibility of the tribological data, Tribology International, 40, 2007, $117-124$.

4. Le Suer, W. M., Patent 2247534, Lubrizol Corp.

5. Cleverley, J. H., Marsh, J. F. and Swietlik, J. M., Patent EP 0323087, Exxon Chemical Corp.

6. Zhou, J., Wu, Z., Zhang, Z., Liu, W. and Xue, Q., Tribological behavior and lubricating mechanism of Cu nanoparticles in oil, Tribology Letters, 8, 2000, 213-218. 
7. Zhang, Z., Xue, Q., Zhang, J., Synthesis, structure and lubricating properties of dialkyldithiophosphate-modifed Mo-S compound nanoclusters, Wear, 209, 1997, 8-12.

8. Zhang, P., Xue, Q., Du, Z. and Zhang, Z., The tribological behavior of LB films of fatty acids and nanoparticles, Wear, 242, 2000, 147-151.

9. Chhowalla, M. and Amaratunga, G. A. J., Thin films of fullerene-like $\mathrm{MoS}_{2}$ nanoparticles with ultra-low friction and wear, Nature, 407, 2000,164-167.

10. Joly-Pottuz, L. and Martin, J. M., unpublished work.

11. Nakagawa, H., Kibi, S., Tagawa, M., Umeno, M. and Ohmae, N., Microtribological properties of ultrathin $\mathrm{C}_{60}$ films grown by molecular beam epitaxy, Wear, 238, 2000, 45-47.

12. Kinoshita, H., Kume, I., Sakai, H., Tagawa, M.and Ohmae, N., High growth rate of vertically aligned carbon nanotubes using a plasma shield in microwave plasma-enhanced chemical vapor deposition, Carbon, 42, 2004, $2735-2777$.

13. Kinoshita, H., Kume, I., Tagawa, M. and Ohmae, N., High friction of a vertically aligned carbon-nanotube film in microtribology, Applied Physics Letters, 85, 2004, 2780-2781.

14. Turq, V., Ohmae, N., Martin, J. M., Fontaine, J., Kinoshita, H. and Loubet, J. L., Influence of humidity on microtribology of vertically aligned carbon nanotube film, Tribology Letters, 19 (1), 2005, 23-28.

15. Miyoshi, K., Street Jr, K. W., Vander Wal, R. L., Andrews, R. and Sayir, A., Solid lubrication by multiwalled carbon nanotubes in air and in vacuum, Tribology Letters, 19 (3), 2005, 191-201.

16. Donnet, C. and Erdemir, A., Historical developments and new trends in tribological and solid lubricant coatings, Surface and Coatings Technology, 180-181, 2004, 76-84.

17. Wang, Y., Lim, S., Luo, J. L. and Xu, Z. H., Tribological and corrosion behaviors of $\mathrm{Al}_{2} \mathrm{O}_{3} /$ polymer nanocomposite coatings, Wear, 260, 2006, 976-983.

18. Chen, W. X., Xu, Z. D., Tenne, R., Rosenstveig, R., Chen, W. L., Gan, H. Y. and Tu, J. P., Wear and friction of Ni-P electroless composite coating including inorganic fullerene- $\mathrm{WS}_{2}$ nanoparticles, Advanced Engineering Materials, 4, 2004, 686-690.

19. Zou, T. Z., Tu, J. P., Zhang, S. C., Chen, L. M., Wang, Q., Zhang, L. L. and Ne, D. H., Friction and wear properties of electroless Ni-P-(IF-MoS 2 ) composite coatings in humid air and vacuum, Materials Science and Engineering A, 426, 2006, 162-168.

20. Katz, A., Redlich, M., Rapoport, L., Wagner, H. D. and Tenne, R., Self-lubricating coatings containing fullerenelike $\mathrm{WS}_{2}$ nanoparticles for orthodontic wires and other possible medical applications, Tribology Letters, 21 (2), 2006, 135-139.

21. Rapoport, L., Fleischer, N. and Tenne, R., Applications of $\mathrm{WS}_{2}\left(\mathrm{MoS}_{2}\right)$ inorganic nanotubes and fullerene-like nanoparticles for solid lubrication and for structural nanocomposites, Journal of Materials Chemistry, 15, 2005, 1782-1788.

22. Rapoport, L., Nepomnyashchy, O., Verdyan, A., Popovitz-Biro, R., Volovik, Y., Ittah, B. and Tenne, R., Polymer nanocomposites with fullerene-like solid lubricant, Avanced Engineering Materials, 6, 2004, 44-48.

23. Rapoport, L., Lvovsky, M., Lapsker, I., Leschchinsky, W., Volovik, Y., Feldman, Y. and Tenne, R., Friction and wear of bronze powder composites including fullerene-like $\mathrm{WS}_{2}$ nanoparticles, Wear, 249, 2001, 150-157.

24. Barrera, E. V., Key methods for developing single-wall nanotube composites, Chemistry and Materials Science, 52 (11), 2007, 38-42.

25. Salvetat, J. P., Mechanical properties of individual nanotubes and composites, Lecture Notes in Physics, 667 , 2006, 439-493.

26. Cai, H., Yan, F. and Xue, Q., Investigation of tribological properties of polyimide/carbon nanotube nanocomposites, Materials Science and Engineering A, 364, 2003, 94-100.

27. Wang, L. Y., Tu, J. P., Chen, W. X., Wang, Y. C., Liu, X. K., Olk, C., Cheng, D. H. and Zhang, X. B., Friction and wear behavior of electroless Ni-based CNT composite coatings, Wear, 254 (12), 2003, 1289-1293.

28. Chen, W. X., Tu, J. P., Wang, L. Y., Gan, H. Y., Xu, Z. D. and Zhang, X. B., Tribological application of carbon nanotubes in a metal-based composite coating and composites, Carbon, 41 (2), 2003, 215-222.

29. Lim, D. S., You, D. H., Choi, H. J., Lim, S. H. and Jang, H., Effect of CNT distribution on tribological behavior of alumina-CNT composites, Wear, 259 (1-6), 2005, 539-544.

30. Sun, J., Gao, L. and Jin, X., Reinforcement of alumina matrix with multi walled carbon nanotubes, Ceramics International, 31 (6), 2005, 893-896.

31. Lim, D.-S., An, J.-W. and Lee, H. J., Effect of carbon nanotube addition on the tribological behavior of carbon/carbon composites, Wear, 252 (5-6), 2002, 512-517. 
32. Schittenhelm, H., Geohegan, D. B. and Jellison, G. E., Synthesis and characterization of single-wall carbon nanotube-amorphous diamond thin-film composites, Applied Physics Letters, 81, 2001, 2097-2099.

33. Kinoshita, H., Kume, I., Sakai, H. and Ohmae, N., Synthesis and mechanical properties of carbon nanotube/diamond-like carbon composite films, Diamond and Related Materials, 16, 2007, 1940-1944.

34. Cabioc'h, T., Jaouen, M., Rivière, J. P., Delafond, J. and Hug, G., Characterization and growth of carbon phases synthesized by high temperature carbon ion implantation into copper, Diamond and Related Materials, 6, 1997, 261-265.

35. Cabioc'h, T., Jaouen, M., Denanot, M. F. and Bechet, P., Influence of the implantation parameters on the microstructure of carbon onions produced by carbon ion implantation, Applied Physics Letters, 73 (21), 1998, 3096-3098.

36. Cabioc'h, T., Jaouen, M., Thune, E., Guérin, P., Fayoux, C. and Denanot, M. F., Carbon onions formation by high-dose carbon ion implantation into copper and silver, Surface and Coatings Technology, 128-129, 2000, $43-50$.

37. Cabioc'h, T., Thune, E., Riviere, J. P., Camelio, S., Girard, J. C., Guérin, P., Jaouen, M., Henrard, L. and Lambin, P., Structure and properties of carbon onion layers onto various substrates, Journal of Applied Physics, 91 (3), $2002,1560-1567$. 
OTE/SPH OTE/SPH

c01 JWBK180-Martin
March 18, 2008

Char Count $=0$ 